\title{
Caracterização do processo de rigor mortis dos músculos Gastrocnemius internus e Fibularis longus de avestruz (Struthio camelus) e da maciez da carne
}

\author{
Characterization of rigor mortis process of muscles \\ Gastrocnemius internus and Fibularis longus of ostrich \\ (Struthio camelus) and meat tenderness
}

Edna Ribeiro dos Santos, ${ }^{*}$ Teófilo José Pimentel da Silva, ${ }^{* *}$ Rogério Tortelly, ${ }^{* *}$ Arlene Gaspar, ${ }^{* \star *}$ Fábio da Costa, ${ }^{*}$ Guilherme Jogaib Jardim****

\begin{abstract}
Resumo
O desenvolvimento do processo de rigor mortis das carcaças de animais de açougue interfere de forma decisiva na qualidade da carne. As características deste processo não foram ainda estabelecidas em carcaças de avestruzes abatidos no Brasil. Foram realizadas análises, nos intervalos de 0,5h, 3,0h, 6,0h, 9,0h, 12,0h e 24,0h após sangria, para a caracterização do processo de rigor mortis nos músculos Gastrocnemius internus e Fibularis longus de seis avestruzes híbridos "Blue neck" e "Red neck", criados em Corumbataí-SP e abatidos em frigorífico sob Inspeção Federal, localizado em Amparo, SP. O tratamento estatístico dos dados demonstrou que durante o resfriamento industrial das carcaças, a temperatura da câmara variou de $11,5^{\circ} \mathrm{C}$ a $-2^{\circ} \mathrm{C}$, enquanto a temperatura dos músculos, de $40,67^{\circ} \mathrm{C}$ a $0,23^{\circ} \mathrm{C}$. O valor do $\mathrm{pH}$ decresceu gradualmente, de 7,05 a 5,97 em Gastrocnemius internus e de 7,26 a 7,14 em Fibularis longus. A contração máxima do sarcômero ocorreu em 0,5 hora, correspondendo ao maior valor de força de cisalhamento e maior perda de peso por cozimento em ambos os músculos. As alterações bioquímicas e estruturais referentes ao processo de rigor mortis em avestruzes corresponderam aos padrões estabelecidos para outras espécies.
\end{abstract}

Palavras-chave: carcaça de avestruz, músculos, rigor mortis, maciez.

\begin{abstract}
The development of the rigor mortis process of carcasses of butcher animals interferes in a decisive way in the meat quality. The characteristics of this process were not established in ostriches carcasses slaughtered in Brazil. Analyses were accomplished, in the intervals of $0.5 \mathrm{~h}, 3.0 \mathrm{~h}, 6.0 \mathrm{~h}, 9.0 \mathrm{~h}, 12.0 \mathrm{~h}$ and $24.0 \mathrm{~h}$ after exsanguination, for the characterization of rigor mortis process in the muscles Gastrocnemius internus and Fibularis longus of six hybrid ostriches "Blue neck" and "Red neck", grown in CorumbataíSP and slaughtered under Federal Inspection, located in Amparo-SP. The statistical treatment of the data demonstrated that during the industrial chilling of the carcasses, the chilly room temperature varied from $11.5^{\circ} \mathrm{C}$ to $-2^{\circ} \mathrm{C}$, while the temperature of the muscles, from $40,67^{\circ} \mathrm{C}$ to $0,23^{\circ} \mathrm{C}$. The value of the $\mathrm{pH}$ decreased gradually, of 7.05 to 5.97 in Gastrocnemius internus and of 7.26 to 7.14 in Fibularis longus. The maximum contraction of sarcomere occurred in 0,5 hour, corresponding to the largest value of shear force and cooking weight loss in both muscles. The biochemical and structural alterations concerning to the rigor mortis process in ostriches corresponded to the established patterns for other species.
\end{abstract}

Keywords: ostrich carcass, muscles, rigor mortis, tenderness.

\section{Introdução}

A estrutiocultura ou criação de avestruzes teve início no Brasil em torno de 1994, apresentando ótima capacidade de adaptação. A carne de avestruz possui propriedades nutricio- nais e sensoriais de elevada qualidade, quando comparada com a bovina e de frango, sendo considerada de consumo crescente, notadamente, por apresentar baixos teores de lipídeos e colesterol (Gianonni, 1996; Paleari et al., 1998).

\footnotetext{
* Programa de Pós-graduação em Medicina Veterinária (Doutorado) - Higiene Veterinária e Processamento Tecnológico de Produtos de Origem Animal, Faculdade de Veterinária, Universidade Federal Fluminense.

** Depto. de Tecnologia dos Alimentos da Faculdade de Veterinária, Universidade Federal Fluminense - Rua Vital Brazil Filho, 64, CEP 24 230340, Niterói, RJ - Brasil.

*** Depto. de Tecnologia de Alimentos da Universidade Federal Rural do Rio de Janeiro.

${ }^{* * * *}$ Discente (Iniciação Científica) - Faculdade de Veterinária, Universidade Federal Fluminense. Autor pra correspondência: Edna Ribeiro dos Santos. E-mail: ednalimentos@ig.com.br
} 
Nas 24 horas após a sangria, inicia-se uma série de transformações bioquímicas e estruturais no tecido muscular, quando o músculo é convertido em carne. Este período é de grande relevância para a qualidade final da carne e dependente de vários fatores que afetam este processo denominado rigor mortis. O declínio do $\mathrm{pH}$ de 6,9 a 7,2 para 5,9 a 6,1 em avestruzes caracteriza-se como uma das alterações post mortem mais significativas, além de ser proporcional ao acúmulo de ácido lático e, inversamente proporcional à reserva de glicogênio da musculatura. O tamanho do sarcômero demonstra uma boa correlação entre seu comprimento e o desenvolvimento do processo de rigor mortis, bem como da maciez de carne (Sales, 1996; Sales e Mellett, 1996; Aberle et al., 2001; Cooper e Horbañczuk, 2002).

O objetivo do presente estudo foi a caracterização do processo de rigor mortis em carcaças de avestruzes e da maciez da carne, através do controle de temperaturas, $\mathrm{pH}$, comprimento do sarcômero, força de cisalhamento e perda de peso por cocção nos músculos Gastrocnemius internus e Fibularis longus durante o resfriamento industrial.

\section{Material e métodos}

Foram escolhidos, aleatoriamente, seis avestruzes machos inteiros híbridos "Blue neck" e "Red neck" no curral de matança do Matadouro Frigorífico Marchiori Ltda (SIF 1504), com 12 a 13 meses de idade e peso vivo entre 89 e $100 \mathrm{~kg}$, criados em Corumbataí, SP, em Amparo, SP. O abate foi realizado conforme as normas descritas no Regulamento da Inspeção Industrial e Sanitária de Produtos de Origem Animal (Brasil, 1997). As carcaças foram pesadas e destinadas à câmara de resfriamento $\left(0,9^{\circ} \mathrm{C}\right.$ à temperatura média do ar e $82,2 \%$ a umidade relativa).

Durante o resfriamento industrial, nos intervalos de $0,5 \mathrm{~h}, 3,0 \mathrm{~h}$, $6,0 \mathrm{~h}, 9,0 \mathrm{~h}, 12,0 \mathrm{~h}$ e $24,0 \mathrm{~h}$ após sangria, foram realizadas mensurações dos parâmetros que caracterizam o processo de rigor mortis.

Para a aferição das temperaturas da câmara de resfriamento e dos músculos foi utilizado um termômetro digital com haste metálica da marca Checktemp. Para a mensuração da temperatura dos músculos, introduziu-se a haste a uma profundidade de $4 \mathrm{~cm}$ em três pontos distintos de cada músculo, tirando-se a média aritmética dos valores.

A determinação do $\mathrm{pH}$ foi realizada em amostras de $10 \mathrm{~g}$ dos músculos Gastrocnemius internus e Fibularis longus com o potenciômetro Handylab 1- Schott, utilizando-se solução homogeneizada com $10 \mathrm{~g}$ da amostra em $100 \mathrm{~mL}$ de água destilada. Foram colhidas amostras dos mesmos músculos com o auxílio de pinça e bisturi, de dimensões de $2,5 \mathrm{~cm}$ de comprimento por $1,5 \mathrm{~cm}$ de largura e $0,5 \mathrm{~cm}$ de espessura, fixados em garras duplas metálicas, evitando alterações nos sarcômeros. As amostras foram identificadas e colocadas em frascos plásticos contendo $250 \mathrm{~mL}$ de formalina tamponada $10 \%$ e transportadas até o laboratório de Tecnologia de Carnes e Derivados da Universidade Federal Fluminense. Após a fixação, as amostras foram clivadas, desidratadas, clarificadas, incluídas em parafina e seccionadas em micrômetro (Pika-Seiko) com espessura de cinco micrômetros. Os cortes histológicos foram corados com
Hematoxilina Fosfotúngstica de Mallory (Behmer et al., 1976). Realizou-se a contagem de 10 sarcômeros em 25 miofibrilas distintas escolhidas ao acaso, através de uma escala dentro de uma ocular milimetrada de $10 \mathrm{~mm}$ acoplada ao microscópio óptico Olimpicus de luz visível em objetiva de imersão, utilizando-se óleo de cedro. As lâminas foram fotografadas com a utilização de máquina acoplada ao microscópio óptico. A média dos valores encontrados foi multiplicada pelo fator de correção da objetiva de imersão de 0,8 mm (Sloss e Kemp, 1978).

Para determinação da força de cisalhamento (maciez), amostras de $200 \mathrm{~g}$, foram pesadas, acondicionadas em saco plástico termorresistente e identificadas. Cozidas em "banhomaria" termostático com circulação de água e controle digital de temperatura, mantendo-se a temperatura em $85^{\circ} \mathrm{C}$, até a temperatura interna de $70^{\circ} \mathrm{C}$; posteriormente, as amostras foram resfriadas e drenadas, pesadas e resfriadas entre $0^{\circ} \mathrm{C}$ e $2^{\circ} \mathrm{C}$. Transportadas em caixas isotérmicas com gelo ao Laboratório de Tecnologia de Carnes e Derivados da Faculdade de Veterinária da Universidade Federal Fluminense (UFF). Procedeu-se a remoção de sete cilindros de $1,27 \mathrm{~cm}$ de diâmetro de carne de cada amostra, sendo cisalhados ao meio no equipamento "Warner-Bratzler Shear Force" modelo 3000, para a obtenção dos valores referentes à força de cisalhamento ou maciez da carne.

Os valores obtidos foram tratados pela análise de variância em fatorial $2^{6}$ para a verificação das influências do tipo de músculo (Gastrocnemius internus e Fibularis longus) e intervalo de tempo post mortem e a interação entre os fatores analisados. Em seguida, foi realizado o teste de comparações múltiplas de Bonferroni ao nível de significância de 5\% (SAS, 1999).

\section{Resultados e discussão}

Através dos dados apresentados na Tabela 1, constatou-se que a temperatura da câmara de resfriamento, comum a todas as carcaças de avestruzes utilizadas nesta pesquisa, decresceu gradativamente de $11,5^{\circ} \mathrm{C}$ para $-2,0^{\circ} \mathrm{C}$, mantendo a temperatura ideal para o desenvolvimento do rigor mortis das carcaças. As temperaturas dos músculos de $40,67^{\circ} \mathrm{C}$ e $40,62^{\circ} \mathrm{C}$, no início do resfriamento passaram a $0,27^{\circ} \mathrm{C}$ e $0,23^{\circ} \mathrm{C}$ em 24 horas no Gastrocnemius internus e Fibularis longus, respectivamente, não diferindo significativamente em nenhum dos intervalos entre músculos. Resultados estes concordantes com os encontrados por Morris et al. (1995) de $40,39^{\circ} \mathrm{C}$ e $3,02^{\circ} \mathrm{C}$ como valores inicial e final de temperatura do músculo Gastrocnemius internus durante esse processo, não diferindo dos valores de outros quatro músculos da coxa (lliofibularis, Iliofemoralis externus, Obturatorius medialis). A diminuição gradativa de temperatura da câmara contribuiu para a qualidade da carne, proporcionando condição básica para que as enzimas proteolíticas atuassem sem o risco de desnaturação das proteínas miofibrilares ou congelamento do músculo antes da conclusão do processo de rigor mortis. Tal fato promoveria reações bioquímicas e características tecnológicas indesejáveis na carne, como textura inadequada, alterações de cor e de capacidade de retenção de água, além de poder potencializar a contaminação microbiológica (Aberle et al., 2001). 
Tabela 1: Valores médios e desvio-padrão $(X \pm s)$ das medidas de temperatura da câmara de resfriamento (TCâmara- ${ }^{\circ} \mathrm{C}$ ), temperatura do músculo $\left(\right.$ TMusc $\left.^{\circ}{ }^{\circ} \mathrm{C}\right)$ e $\mathrm{pH}$ dos músculos Gastrocnemius internus (Gi) e Fibularis longus (FI) em seis (n) avestruzes nos seis diferentes intervalos de tempo (h) após sangria no Matadouro Frigorífico Marchiori Ltda. (Amparo-SP)

\begin{tabular}{|c|c|c|c|c|c|c|c|c|}
\hline \multirow{2}{*}{ Medidas } & \multirow{2}{*}{ Amostras } & \multirow{2}{*}{$\mathrm{n}$} & \multicolumn{6}{|c|}{ Tempo após sangria (horas) } \\
\hline & & & 0,5 & 3 & 6 & 9 & 12 & 24 \\
\hline Temperatura & Câmara & 6 & $\begin{array}{c}11,5^{\mathrm{a}} \\
( \pm 0,06)\end{array}$ & $\begin{array}{c}1,5^{\mathrm{b}} \\
( \pm 0,11)\end{array}$ & $\begin{array}{c}0,90^{c} \\
( \pm 0,06)\end{array}$ & $\begin{array}{c}-0,50^{d} \\
( \pm 0,09)\end{array}$ & $\begin{array}{c}-0,20^{\mathrm{e}} \\
( \pm 0,06)\end{array}$ & $\begin{array}{c}-2,00^{\dagger} \\
( \pm 0,06)\end{array}$ \\
\hline & $\begin{array}{c}\mathrm{Gi} \\
(\mathrm{X} \pm \mathrm{s})\end{array}$ & 6 & $\begin{array}{l}40,67^{\mathrm{aA}} \\
( \pm 0,96)\end{array}$ & $\begin{array}{l}17,43^{\mathrm{bA}} \\
( \pm 1,63)\end{array}$ & $\begin{array}{c}9,57^{\mathrm{CA}} \\
( \pm 1,25)\end{array}$ & $\begin{array}{c}5,45^{\mathrm{dA}} \\
( \pm 0,36)\end{array}$ & $\begin{array}{c}2,98^{\mathrm{eA}} \\
( \pm 0,22)\end{array}$ & $\begin{array}{c}0,27^{\mathrm{tA}} \\
( \pm 0,05)\end{array}$ \\
\hline Temperatura & $\begin{array}{c}F I \\
(X \pm s)\end{array}$ & 6 & $\begin{array}{l}40,62^{\mathrm{aA}} \\
( \pm 0,94)\end{array}$ & $\begin{array}{l}17,32^{\mathrm{bA}} \\
( \pm 1,54)\end{array}$ & $\begin{array}{c}9,53^{\mathrm{cA}} \\
( \pm 1,22)\end{array}$ & $\begin{array}{c}5,42^{\mathrm{dA}} \\
( \pm 0,33)\end{array}$ & $\begin{array}{c}2,95^{\mathrm{eA}} \\
( \pm 0,96)\end{array}$ & $\begin{array}{c}0,23^{\mathrm{fA}} \\
( \pm 0,05)\end{array}$ \\
\hline $\mathrm{pH}$ & $\begin{array}{c}\mathrm{Gi} \\
(\mathrm{X} \pm \mathrm{s}) \\
\mathrm{FI} \\
(\mathrm{X} \pm \mathrm{s}) \\
\end{array}$ & $\begin{array}{l}6 \\
6\end{array}$ & $\begin{array}{c}7,05^{\mathrm{aB}} \\
( \pm 0,07) \\
7,26^{\mathrm{aA}} \\
( \pm 0,12) \\
\end{array}$ & $\begin{array}{c}6,51^{\mathrm{bB}} \\
( \pm 0,08) \\
6,74^{\mathrm{bA}} \\
( \pm 0,06) \\
\end{array}$ & $\begin{array}{c}6,14^{\mathrm{cB}} \\
( \pm 0,07) \\
6,28^{\mathrm{cA}} \\
( \pm 0,04) \\
\end{array}$ & $\begin{array}{c}6,08^{\mathrm{CA}} \\
( \pm 0,09) \\
6,21^{\mathrm{cA}} \\
( \pm 0,05) \\
\end{array}$ & $\begin{array}{c}6,07^{\mathrm{CA}} \\
( \pm 0,05) \\
6,17^{\mathrm{CA}} \\
( \pm 0,07) \\
\end{array}$ & $\begin{array}{c}5,97^{\mathrm{CB}} \\
( \pm 0,12) \\
6,14^{\mathrm{CA}} \\
( \pm 0,07) \\
\end{array}$ \\
\hline
\end{tabular}

a,b,c,d,e Médias na mesma linha seguidas de diferentes letras minúsculas diferem significativamente quanto ao intervalo de tempo após sangria $(p<0,05)$

A, B Médias na mesma coluna seguidas de diferentes letras maiúsculas diferem significativamente quanto ao músculo $(p<0,05)$

É comprovado que o armazenamento post mortem em temperatura de refrigeração resulta na melhoria significativa da maciez proporcionada pela degradação das miofibrilas através de enzimas proteolíticas endógenas (catepsinas e calpaínas) do músculo. A velocidade do rigor mortis é controlada, principalmente, por características do tecido muscular como teor de reserva de glicogênio, $\mathrm{pH}$ e temperatura (Aberle et al., 2001).

$\mathrm{O}$ pH inicial de 7,05 em Gastrocnemius internus diferiu significativamente $(\mathrm{p}<0,05)$ até as 6 horas, quando atingiu valor de 6,14 , não havendo diferença significativa até as 24 horas após sangria, quando se estabeleceu a resolução do rigor mortis em pH 5,97. Em relação ao Fibularis longus, a queda do $\mathrm{pH}$ foi significativa até as seis horas, correspondendo a 6,28 e 6,14 às 24 horas, portanto, se estabeleceu também em torno de seis horas após a sangria, correspondendo a valores significativamente maiores $(p<0,05)$ nos mesmos intervalos de tempo quando comparado ao Gastrocnemius internus. Nos mesmos intervalos (6 e 24 horas post mortem) Sales e Mellett (1996) encontraram valores de 6,12 e 6,05 para Gastrocnemius internus. Os valores finais de $\mathrm{pH}$, encontrados para os dois músculos, estão em concordância com os citados por Paleari et al. (1998) que afirmaram ser o $\mathrm{pH}$ da carne de avestruz maior
Tabela 2: Valores médios e desvio-padrão $(X \pm s)$ das medidas de comprimento do sarcômero (CS- $\mu \mathrm{m})$, força de cisalhamento (FC-Kg) e perda de peso por cozimento (PC-\%) dos músculos Gastrocnemius internus (Gi) e Fibulares longus (FI) encontrados em seis (n) avestruzes nos seis diferentes intervalos de tempo (h) após sangria no Matadouro Frigorífico Marchiori Ltda. (Amparo-SP)

\begin{tabular}{|c|c|c|c|c|c|c|c|c|}
\hline \multirow{2}{*}{\multicolumn{2}{|c|}{$\begin{array}{l}\text { Medidas } \\
\text { Amostras }\end{array}$}} & \multirow{2}{*}{$\mathrm{n}$} & \multicolumn{6}{|c|}{ Tempo após sangria (h) } \\
\hline & & & 0,5 & 3 & 6 & 9 & 12 & 24 \\
\hline CS & $\begin{array}{l}\mathrm{Gi} \\
\mathrm{FI}\end{array}$ & 6 & $\begin{array}{l}1,60^{\mathrm{aA}} \\
(0,02) \\
1,50^{\mathrm{a}} \\
(0,01) \\
\end{array}$ & $\begin{array}{l}1,78^{\mathrm{bA}} \\
(0,02) \\
1,65^{\mathrm{bB}} \\
(0,02)\end{array}$ & $\begin{array}{l}1,80^{\mathrm{CA}} \\
(0,01) \\
1,67^{\mathrm{CB}} \\
(0,02) \\
\end{array}$ & $\begin{array}{l}1,80^{\mathrm{CA}} \\
(0,02) \\
1,71^{\mathrm{CB}} \\
(0,02) \\
\end{array}$ & $\begin{array}{l}1,85^{\mathrm{dA}} \\
(0,03) \\
1,70^{\mathrm{CB}} \\
(0,02) \\
\end{array}$ & $\begin{array}{l}1,94^{\mathrm{eA}} \\
(0,03) \\
1,76^{\mathrm{cB}} \\
(0,02)\end{array}$ \\
\hline $\mathrm{FC}$ & $\begin{array}{l}\mathrm{Gi} \\
\mathrm{FI}\end{array}$ & 6 & $\begin{array}{c}10,78^{\mathrm{aB}} \\
(0,60) \\
13,14^{\mathrm{AA}} \\
(0,88) \\
\end{array}$ & $\begin{array}{l}6,75^{\mathrm{bB}} \\
(0,75) \\
8,54^{\mathrm{bA}} \\
(0,59)\end{array}$ & $\begin{array}{l}4,91^{\mathrm{bB}} \\
(0,53) \\
6,00^{\mathrm{bA}} \\
(0,58)\end{array}$ & $\begin{array}{l}4,18^{\mathrm{bB}} \\
(0,55) \\
5,20^{\mathrm{bA}} \\
(0,69)\end{array}$ & $\begin{array}{l}3,62^{\mathrm{CB}} \\
(0,50) \\
4,49^{\mathrm{CA}} \\
(0,54)\end{array}$ & $\begin{array}{l}2,78^{\mathrm{dB}} \\
(0,57) \\
3,52^{\mathrm{dA}} \\
(0,57)\end{array}$ \\
\hline $\mathrm{PC}$ & $\begin{array}{l}\mathrm{Gi} \\
\mathrm{FI}\end{array}$ & $\begin{array}{l}6 \\
6\end{array}$ & $\begin{array}{c}30,33^{\mathrm{aB}} \\
(0,36) \\
34,22^{\mathrm{aA}} \\
(0,23)\end{array}$ & $\begin{array}{c}26,47^{\mathrm{bB}} \\
(0,38) \\
29,28^{\mathrm{bA}} \\
(0,28)\end{array}$ & $\begin{array}{c}26,07^{\mathrm{cB}} \\
(0,21) \\
28,93^{\mathrm{bA}} \\
(0,38)\end{array}$ & $\begin{array}{c}25,70^{\mathrm{dB}} \\
(0,47) \\
28,33^{\mathrm{CA}} \\
(0,29)\end{array}$ & $\begin{array}{c}25,27^{\mathrm{dB}} \\
(0,38) \\
28,12^{\mathrm{dA}} \\
(0,23)\end{array}$ & $\begin{array}{c}24,83^{\mathrm{eB}} \\
(0,28) \\
26,83^{\mathrm{dA}} \\
(0,26)\end{array}$ \\
\hline
\end{tabular}

a,b,c,d,e Médias na mesma linha seguidas de diferentes letras minúsculas diferem significativamente quanto ao intervalo de tempo após sangria $(p<0,05)$

A, B Médias na mesma coluna seguidas de diferentes letras maiúsculas diferem significativamente quanto ao músculo $(p<0,05)$. 
De acordo com os valores apresentados na Tabela 2, ocorreu a contração máxima do sarcômero em $0,5 \mathrm{~h}(1,60 \mu \mathrm{m})$, aumentando até as 24 horas após sangria para 1,94 $\mu \mathrm{m}$ em Gastrocnemius internus e de $1,50 \mu \mathrm{m}$ para $1,76 \mu \mathrm{m}$, nos mesmos intervalos, para o Fibularis longus. Os valores encontrados na $3^{\text {a }}$ hora post mortem em diante revelaram aumento gradativo até a última medição (24므 hora). Ao alcançar a temperatura em torno de $10^{\circ} \mathrm{C}$ às 6 horas post mortem e a contração máxima do sarcômero em $0,5 \mathrm{~h}$, pode-se supor que seria improvável a ocorrência do encurtamento pelo frio em carcaças de avestruzes, constituindo perda de qualidade da carne, em comparação com carcaças de bovinos. O músculo Gastrocnemius internus apresentou valores de comprimento do sarcômero significativamente maiores $(p<0,05)$ que 0 Fibularis longus, em todos os intervalos de tempo após sangria. Por sua vez, Sales (1996) encontrou valor de 2,34 mm para comprimento do sarcômero de Gastrocnemius internus e média de 2,04 mm em seis músculos de avestruzes em 24h post mortem, cujos resultados são superiores aos mensurados neste estudo, provavelmente devido a utilização da técnica de microscopia de contraste de fase sem fixação da amostra. $\mathrm{O}$ comprimento do sarcômero sofre alterações durante o período do rigor mortis, devido à ação enzimática, na medida em que progride sua resolução (ABERLE et al., 2001).

A Figura 1 ilustra as diferenças ocorridas na microestrutura muscular desde o início do processo de rigor mortis.

No início das medidas (0,5h após sangria) observou-se a integridade e proximidade entre duas linhas Z (Figura 1a, c), correspondendo ao comprimento do sarcômero (Tabela 2), significativamente maior ao final de 24 horas (Figura 1b, d). Portanto, torna-se nítida a ação das enzimas proteolíticas, calpaínas e catepsinas, determinando a resolução deste processo bioquímico. Além da degradação das proteínas titina e nebulina que conferem suporte para as principais proteínas miofibrilares: actina e miosina (Jaarsveld et al., 1997). As calpaínas, por atuarem principalmente em $\mathrm{pH}$ considerados elevados, teriam iniciado o processo de resolução, como as responsáveis pela hidrólise das proteínas titina, nebulina e, em menor proporção, das cabeças de miosina. As catepsinas, liberadas para o sarcoplasma após a desestruturação da membrana lisossomal, devido ao $\mathrm{pH}$ mais ácido, provavelmente, fragmentaram as proteínas troponina e a tropomiosina.

Na Figura 2 apresenta-se uma comparação entre as médias relativas ao comprimento do sarcômero e força de cisalhamento de ambos os músculos analisados.

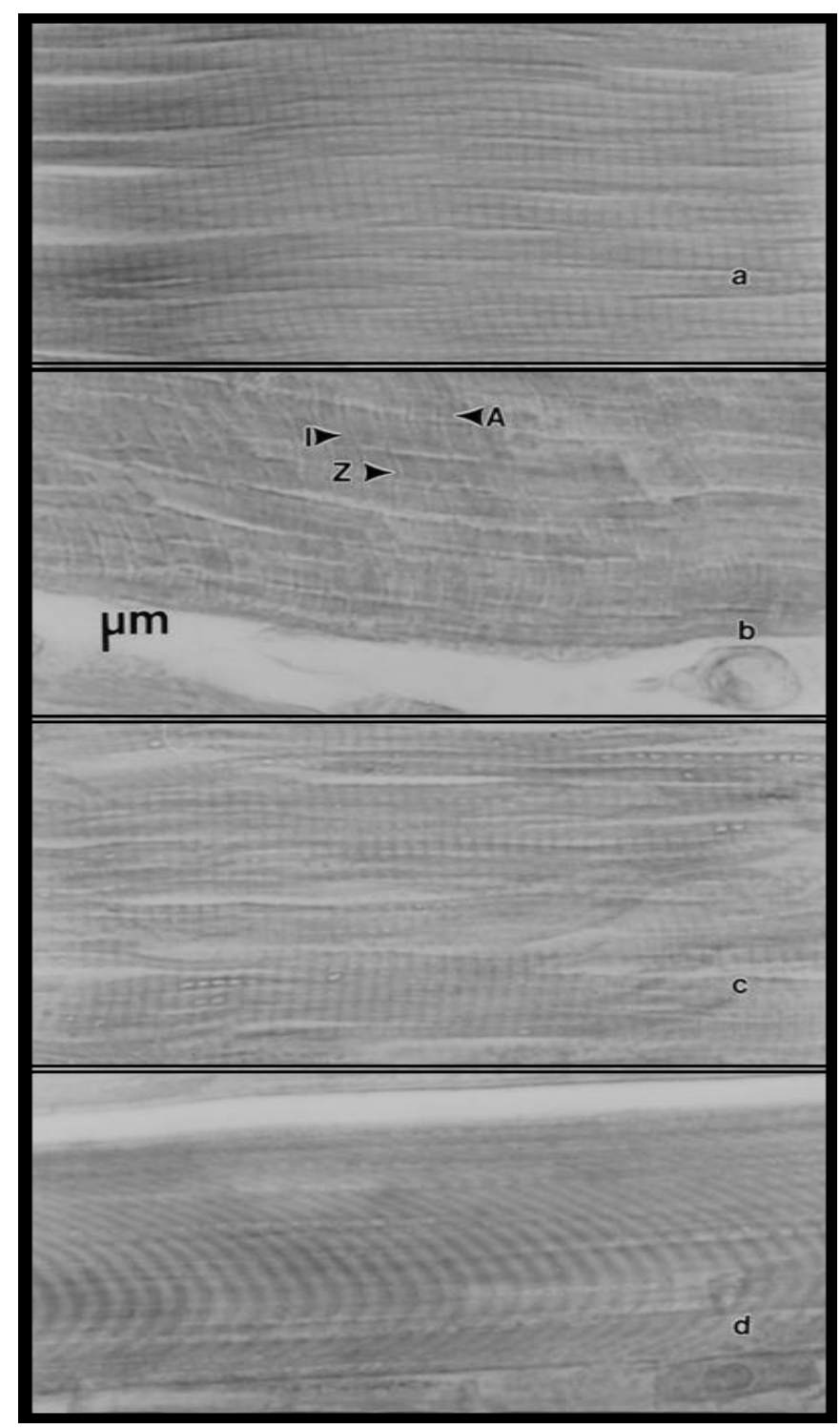

Figura 1: Fotomicrografias ópticas do músculo Gastrocnemius internus (Gi) e Fibularis longus (FI) durante o resfriamento industrial, mostrando as bandas A, I e disco Z (aumento de 1000 vezes), fixado com formalina tamponada neutra e corado com hematoxilina fosfotúngstica de Mallory: a) músculo Gi aos $0,5 \mathrm{~h}$ após a sangria e b) na $24^{\mathrm{a}}$ hora após a sangria; c) músculo $\mathrm{FI}$ aos $0,5 \mathrm{~h}$ após a sangria e d) na $24^{\mathrm{a}}$ hora após a sangria.

Ao comparar os valores da força de cisalhamento e do comprimento do sarcômero, observou-se que a contração máxima do sarcômero e a maior força de cisalhamento ocorreram no mesmo estágio de rigor mortis, portanto, 0,5h após sangria, em ambos os músculos, concordando com a influência do comprimento do sarcômero na maciez da carne. De acordo com os resultados encontrados, o músculo Gastrocnemius internus originou, ao

Figura 2: Médias referentes ao comprimento do sarcômero (CS) e força de cisalhamento (FC) dos músculos Gastrocnemius internus (Gi) e Fibularis longus (FI) de avestruz, nos intervalos de 0,5, 3,0, 6,0, 9,0, 12,0, 24,0 horas após sangria).

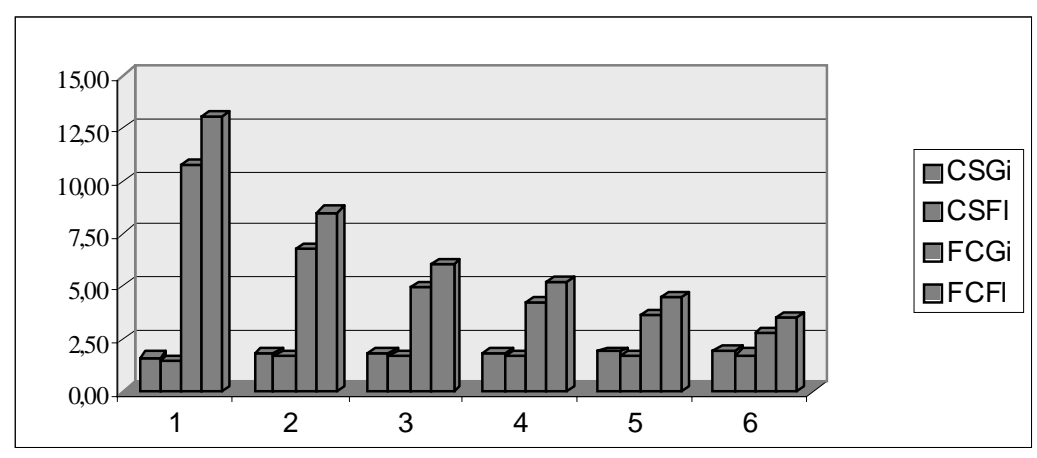


final do processo de resfriamento, carne mais macia que o Fibularis longus. Os valores de força de cisalhamento foram de $2,78 \mathrm{~kg}$ e $3,52 \mathrm{~kg}$ para Gastrocnemius internus e Fibularis longus, respectivamente. Diferença concordante com valores encontrados por Cooper e Horbañczuk (2002) de 2 kg e 3,5 kg para Gastrocnemius internus e Fibularis longus, nesta ordem, enquanto Sales (1996) encontrou 2,97 kg para Gastrocnemius internus e média de $3,35 \mathrm{~kg}$ para a força de cisalhamento de seis músculos de avestruz. Complementando, Hoffman e Fisher (2001) citaram força de cisalhamento de 4,4 kg, para a carne de avestruz, sem distinção do corte ou músculo. Esses valores demonstraram que a carne de avestruz pode ser considerada macia, pois de acordo com classificações preestabelecidas, como a de Jeremiah et al. (1991), o limite corresponde a $5 \mathrm{~kg}$ o valor para que a carne bovina seja considerada macia.

\section{Referências}

ABERLE, E.D.; FORREST, J.C.; GERRARD, D.E.; EDWAR, W. M. Principles of Meat Science. 4. ed. Lowa: Kendall//Hunt Publishing Company, 2001, $354 \mathrm{p}$.

BEHMER, O. A.; TOLOSA, E. M. C.; NETO, A. G. F. Manual de Técnicas para Histologia Normal e Patológica. São Paulo: EDART-Editora USP, 1976, $236 \mathrm{p}$.

BRASIL, Ministério da Agricultura e do Abastecimento. Secretaria Nacional de Defesa Agropecuária. Regulamento de Inspeção Industrial e Sanitária de Produtos de Origem Animal (Aprovado pelo Decreto ${ }^{\circ}$ 30.691 de 29-03-52, alterado pelos Decretos nr. 1,255 de 25-06-62, 1.236 de 02-09-94, $n^{\circ} 1.812$ de 08-02-96 e n².244 de 05-06-97). DIPOA-MAPA, Brasília, DF, 1997, 241 p.

COOPER, G. R.; HORBAÑCZUK, J. O. Anatomical and physiological characteristics of ostrich (Struthio camelus var. domesticus) meat determine its nutritional importance for man. Animal Science Journal, v. 73, p. 1667-173, 2002.

GIANONNI, M. L. Criação de avestruzes: a situação brasileira. Conferência Apinco 96 de Ciência e Tecnologia Avícolas, p. 7-16, 1996.

HOFFMAN, L. C.; FISHER, P. Comparison of meat quality characteristics between young and old ostriches. Meat Science, v. 59, n. 3, p. 35-337, 2001.

JAARSVELD, F. P.V.; NAUDÉ, R. J.; OELOFSEN, W. The effects of Ca ions, EGTA and storage time on myofibrillar protein degradation, levels

\section{Conclusões}

O declínio da temperatura das carcaças de avestruzes durante o resfriamento industrial ocorreu dentro dos padrões preestabelecidos; não diferindo entre os dois músculos. O valor do $\mathrm{pH}$ diminuiu, estabelecendo-se em 6 horas após a sangria até as 24 horas, sendo significativamente maior no músculo Fibularis longus. A contração máxima do rigor mortis, caracterizado pelo menor comprimento do sarcômero e maior força de cisalhamento, se estabeleceu em $0,5 \mathrm{~h}$ após a sangria; sendo maior no músculo Fibularis longus. A força de cisalhamento foi inversamente proporcional ao comprimento do sarcômero, sendo maior no músculo Fibularis longus, portanto o Gastrocnemius internus foi considerado o mais macio.

of $\mathrm{Ca}^{+2}$-dependent proteases and cathepsins $B, H, L$ and $D$ of ostrich skeletal muscle. Meat Science, v. 45, n. 4, p. 517-529, 1997.

JEREMIAH, L. E.; TONG, A. K. Y.; GIBSON, L. L. The insefulness of muscle color and $\mathrm{pH}$ for segregating beef carcasses into tenderness groups. Meat Science, v. 30, p. 97-114, 1991.

MORRIS, C. A.; HARRIS, S. D.; MAY, S. G.; JACKSON, T. C.;HALE, D. S.;MILLER, R. K.; KEETON, J. T.; ACUFF, G. R.; LUCIA, L. M.; SAVELL, J. W. Ostrich slaughter and fabrication: 1 . Slaughter yields of carcass and effects of electrical stimulation on post-mortem $\mathrm{pH}$. Poultry Science, v. 74, p. 1683-1687, 1995.

PALEARI, M. A.; CAMISASCA, S.; BERETTA, G.; RENON, P.; CORSICO, P.; BERTOLO, G.; CRIVELLI, G. Ostrich Meat: Physico-chemical characteristics and comparison with turkey and bovine meat. Meat Science, v. 48, n. 3-4, p. 205-210, 1998.

SALES, J. Histological, biophysical, physical and chemical characteristics of different ostrich muscles. Journal of Food Agriculture, v. 70, p. 109114, 1996.

SALES, J.; MELLETT, F. D. Post- mortem pH Decline in Different Ostrich Muscles. Meat Science, v. 42, n. 2, p. 235-238, 1996.

SAS Intitute. SAS ${ }^{R}$ User's Guide. 6.04 Edition. SAS Institute Inc., Cary, NC. 1999.

SLOSS, M. W. B. S.; KEMP, R. L. A. B. Veterinary clinical parasitology. 5 ed. Ames: Lowa State University Press, 1978. 247 p. 